\title{
A Quantitative Investigation of Inertial Power Harvesting for Human-powered Devices
}

\author{
Jaeseok Yun ${ }^{\dagger}$, Shwetak Patel ${ }^{\ddagger}$, Matt Reynolds ${ }^{\S}$, Gregory Abowd ${ }^{\dagger}$ \\ $\dagger$ School of Interactive \\ Computing \& GVU Center \\ College of Computing \\ Georgia Institute of \\ Technology \\ Atlanta, GA 30332 USA \\ \{jaeseok,abowd\}@cc.gatech.edu \\ $\ddagger$ Computer Science \& \\ Engineering \\ Electrical Engineering \\ University of Washington \\ Seattle, WA 98195 USA \\ shwetak@cs.washington.edu \\ $\S$ Electrical \& Computer \\ Engineering \\ Pratt School of Engineering \\ Duke University \\ Durham, NC 27708 USA \\ matt.reynolds@duke.edu
}

\begin{abstract}
We present an empirical study of the long-term practicality of using human motion to generate operating power for body-mounted consumer electronics and health sensors. We have collected a large continuous acceleration dataset from eight experimental subjects going about their normal daily routine for 3 days each. Each subject is instrumented with a data collection apparatus that simultaneously logs 3 -axis, $80 \mathrm{~Hz}$ acceleration data from six body locations. We use this dataset to optimize a first-principles physical model of the commonly used velocity damped resonant generator (VDRG) by selecting physical parameters such as resonant frequency and damping coefficient to maximize harvested power. Our results show that with reasonable assumptions on size, mass, placement, and efficiency of VDRG harvesters, most body-mounted wireless sensors and even some consumer electronics devices, may be powered continuously and indefinitely from everyday motion.
\end{abstract}

\section{ACM Classification Keywords}

C.5.3 Microcomputers: Portable devices

\section{General Terms \\ Human Factors, Experimentation}

\section{Author Keywords \\ Inertial power harvesting, human-powered devices}

\section{INTRODUCTION}

Power remains a key to unlocking the potential for a sustainable ubicomp reality, particularly for body-worn mobile electronics. Paradiso and Starner show the promise of scavenging energy to power mobile electronics [20, 22, $28,29]$. While power harvesting from natural and renewable sources has been a longstanding research area, there is surprisingly little in the published literature that

Permission to make digital or hard copies of all or part of this work for personal or classroom use is granted without fee provided that copies are not made or distributed for profit or commercial advantage and that copies bear this notice and the full citation on the first page. To copy otherwise, or republish, to post on servers or to redistribute to lists, requires prior specific permission and/or a fee.

UbiComp'08, September 21-24, 2008, Seoul, Korea.

Copyright 2008 ACM 978-1-60558-136-1/08/09...\$5.00. demonstrates the long-term, practical capabilities of garnering energy from daily human activity. Human activity levels range greatly over the course of a typical day, including periods of sleep, quiet intervals for reading or office work, and highly active periods of walking, running, or participating in sports activities. Most prior research into power harvester performance using human subjects has been performed for short time intervals in laboratory simulations of selected human activities. In this work we report the first all-day, continuous all-activity study of inertial power harvester performance using eight untethered human subjects going about their ordinary lives.

We have created an untethered, wearable apparatus for gathering calibrated 3 -axis acceleration data continuously at $80 \mathrm{~Hz}$ sampling rate, from 6 different body locations. We collected datasets spanning 24 hours continuous collection periods, and obtained three days of ordinary human activity from eight experimental participants. This unique, high-fidelity dataset provides the input for our power generation experiments. We next developed a firstprinciples numerical model using MATLAB Simulink of the most common type of inertial power harvester, the Velocity Damped Resonant Generator (VDRG), that provides an accurate and realistic estimate of achievable performance from an inertial energy harvester based on the VDRG architecture. For common wireless health sensors and several commonly used consumer electronics devices (such as GPS receivers, cell phones, and MP3 decoders), we used reasonable assumptions on form factor, placement and efficiency of realizable harvesters to create models of appropriate VDRGs that could be built into each device. By feeding each subject's acceleration dataset into our VDRG models, we estimate available power to indicate which electronic devices or wireless sensors can be powered continuously and indefinitely from energy harvested over the course of a typical day's activity. We explore 6 different locations for placement of the harvester-powered device, considering devices appropriate for each location on the body.

Our results are particularly exciting because they are based on measured sensor data gathered with high fidelity from everyday activities. The proposed inertial harvester designs - given in terms of the critical features of form factor, proof mass, resonant frequency and damp- 
ing coefficient - are currently optimized on a per individual basis and do not yet generalize across the entire subject pool. However, we discuss the remaining challenges for establishing general design parameters for body-mounted motion-powered generators. We can now conclude with confidence that continuously-operating $\mathrm{m}$ otion-powered wireless health sensors are entirely within the realm of practical feasibility and that activity-specific inertial power harvesting will provide continuous operating power for some commonly-used consumer electronics devices. We expect that with the refinement of a new class of inertial power harvesters, called adaptively tuned velocity damped resonant generators (AT-VDRGs) we can move beyond activity-specific power harvesters to devices that will extract energy nearly optimally over most human activities.

\section{RELATED WORK}

The focus of considerable study, energy harvesting has a long history. More recently, Paradiso and Starner presented an extensive survey of the various available energy sources to power mobile devices [20,22, 29], including solar power [17], background radio signals [33], thermal gradients [30], vibrational excitation [11], and humans. Paradiso's team at MIT has demonstrated some clever means of exploiting human motion, such as a selfpowered, spring-loaded igniter switch capable of wirelessly transmitting a static ID number [21] and a shoemounted piezoelectric that produces enough energy to power a wireless transmitter [27].

As these examples suggest, and summarized by Starner [28], the human body is a tremendous energy reservoir. Our interest is in the use of human motion to generate energy. The first use of human-induced vibration as a practical energy source was the self-winding pedometer watch created in the 1600s [14]. Self-winding watches exploited human motion to wind the mechanism, yielding enough power to operate the watch. Since that time, several commercial products using human-induced vibration have been invented [22]. Examples include a shakable flashlight, which employs electro-magnetic induction of a magnet moving through electrical coils to power lights, and hand cranks, which have also been integrated into small flashlights and radios to produce temporary power. Similarly, machine-induced vibration can also be exploited to harvest electrical power $[5,6]$. Harvesters are resonantly tuned to a particular frequency generated from motors or vehicles, yielding electrical power ranging from $1 \mathrm{~mW}$ to $45 \mathrm{~mW}$, depending upon the acceleration magnitude, easily powering industrial wireless sensors without batteries. This capability of frequencytuned harvesting provided the motivation for this research into the feasibility of a macro-size power harvester using human-induced vibration.

Unobtrusively scavenging energy from daily human activity is a very attractive idea in human-powered wearable computing. However, few studies have examined closely how human-induced vibration can be used for scavenging energy and how much power can practically be scavenged from vibrational energy in daily ordinary human motion. Amirtharajah et al. modeled human walking as a stochastic signal with a narrowband power spectral density centered on $2 \mathrm{~Hz}$. Their model estimated that $400 \mu \mathrm{W}$ of power can be generated using human walking as a vibrational source [11]. Mitcheson et al. presented optimized architectures for vibration-driven micropower generators with a sinusoidal driving motion [19]. However, human motion is a complex combination of sinusoidal components, which are difficult to harvest simultaneously. Büren et al. measured acceleration from nine locations on the bodies of walking human subjects and estimated the maximum output power using simulation models of inertial microgenerators [13]. The result of simulations showed that the maximum power density is around $2.1 \mathrm{~mW} / \mathrm{cm}^{3}$. However, these simulations were performed with acceleration signals measured from standard walking motion for 60 seconds on a treadmill running at a constant $4 \mathrm{~km} / \mathrm{h}$, and there is a question of how this result would extrapolate to normal daily activity, which is what we want to explore. Moreover, that work did not account for the expected power efficiency (useful electrical power/simulated output power) of the harvester model.

Rome et al. exploited a vertical excursion of the 20-38 $\mathrm{Kg}$ load in a rigid backpack during normal walking and climbing on an inclined treadmill [24]. They showed that users were able to generate up to $7.4 \mathrm{~W}$ of power, depending upon the mass of the load. Despite the attractive power harvested from the backpack, the huge mass of $38 \mathrm{Kg}$ will limit its use to the niche market such as adventurers or military personnel. Recently, Kuo's team developed a knee brace generator that produced an average of $5 \mathrm{~W}$ of electricity with one device mounted on each leg [15]. However, the user has to wear a bulky and heavy $(1.8 \mathrm{Kg})$ orthopedic knee brace around the knee, so it is difficult for the harvester to be embedded into the real objects.

For a self-sustaining system, Tröster's team created a button-sized solar-powered node composed of sensors, a processor, RF transmitter, and solar cells, to collect, process, and forward sensory data to a central wearable device in [12]. They showed the possibilities of energy harvesting through ordinary exposure to sunlight and indoor light for an entire day. This style of energy harvesting through everyday activities echoes our motivation in this paper.

\section{UNDERSTANDING OF WEARABLE DEVICES}

Given the power garnered from humans, we are able to imagine powering devices such as consumer electronics and self-sustaining body sensor networks. The tapped power can be an alternative to batteries for consumer electronics such as watches, MP3 players, cell phones, and GPS receiver kits. Another possible application of human power harvesting is to power wearable sensors for monitoring human vital signs. Monitoring of the status of human health could play a key role in the future health care services for disease prevention as well as the treatment for chronically ill patients. The required power for all electronics has been tabulated and presented in Table 1. We chose ultra-low power-consuming electronics and only considered powering core components, e.g., an MP3 
Table 1. Required power for components in consumer mobile devices and health sensors.

\begin{tabular}{|c|c|c|}
\hline Electronics & Reference & Required Power \\
\hline MP3 decoder chip & {$[10]$} & $58 \mathrm{~mW}$ \\
\hline $\mathrm{RF}$ receiver chip & {$[2]$} & $24 \mathrm{~mW}$ \\
\hline GPS receiver chip & 9 & $15 \mathrm{~mW}$ \\
\hline 6D motion sensor & 25 & $14.4 \mathrm{~mW}$ \\
\hline Cell phone (standby) & [8] & $8.1 \mathrm{~mW}$ \\
\hline PPG sensor $^{a}$ & 23 & $1.473 \mathrm{~mW}$ \\
\hline Humidity & [4] & $1 \mathrm{~mW}$ \\
\hline Pressure & $16]$ & $0.5 \mathrm{~mW}$ \\
\hline 3D accelerometer & {$[1]$} & $0.324 \mathrm{~mW}$ \\
\hline Temperature & [3] & $27 \mu \mathrm{W}$ \\
\hline Wristwatch & [7] & $7 \mu \mathrm{W}$ \\
\hline Memory $\mathrm{R} / \mathrm{W}^{b}$ & [18] & $2.17 \mu \mathrm{W}$ \\
\hline A-D conversion & $26]$ & $1 \mu \mathrm{W}$ \\
\hline $\mathrm{RF}$ transmission ${ }^{c}$ & 32 & sub $\mu \mathrm{W}$ \\
\hline
\end{tabular}

${ }^{a}$ Photoplethysmograph sensor, that measures the blood pressure, the heart rate, and the respiration rate.

${ }^{b}$ With $1 \mathrm{Kbit} / \mathrm{s}, 1 \mathrm{~m}$ distance data transmission

${ }^{c}$ With $1 \mathrm{Kbit} / \mathrm{s}, 1 \mathrm{~m}$ distance data transmission.

decoder chip, but not an LCD display or a headphone for an MP3 player. Thus, the main result of this paper is to demonstrate whether the power garnered from daily human motion can practically power the low-power components for wearable electronics in Table 1 in terms of real objects and the body locations.

\section{POWER HARVESTER MODEL}

In order to use the measured acceleration dataset to estimate achievable electrical power output, we have developed a model for the power harvester in MATLAB Simulink, a numerical simulation program. We are concerned with the energy available from human body motion, so we concentrate on inertial generator models where the body's acceleration imparts forces on a proof mass whose motion is coupled to the generator itself. Mitcheson classified inertial generators into three main categories: the Velocity-Damped Resonant Generator (VDR$\mathrm{G})$, the Coulomb-Damped Resonant Generator (CDRG), and the Coulomb-Force Parametric Generator (CFPG), depending on whether their operating principles are electromagnetic, electrostatic, or piezoelectric [19]. It has been shown that when the internal displacement travel of a generator exceeds $0.5 \mathrm{~mm}$, e.g., for macro-scale (nonMEMS) generators, the VDRG is the recommended architecture [13]. We have therefore concentrated on the VDRG model for our simulations.

Vibration-driven generators are ideally represented as a damped mass-spring system, that is, a second-order differential equation, as in Equation 1:

$$
m \ddot{z}(t)=-K z(t)-D \dot{z}(t)-m \ddot{y}(t),
$$

where $m$ is the value of the proof mass, $K$ is the spring constant, $D$ is the damping coefficient, $y(t)$ is the displacement of the generator, $z(t)$ is the relative displacement between the proof mass and the generator, and $t$ is time [31]. In this damped mass-spring system, the electrical energy generated is represented as the energy

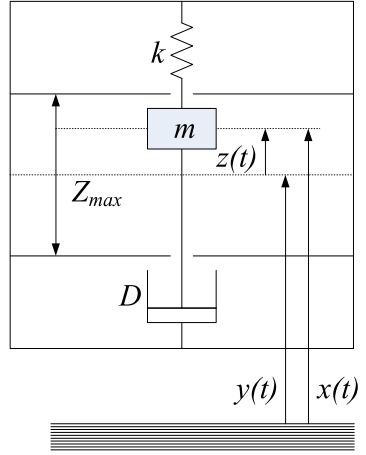

Figure 1. A generic model for the velocity-damped resonant generator.

dissipated in the mechanical damper. In an electromagnetic generator, this damping is due to Lenz's law as the moving magnet does mechanical work when traveling in and out of the generator's coils.

A general model and operating principle for the VDRG is shown in Figure 1. Once the generator is accelerated with displacement $y(t)$, the inertia of the proof mass $m$ causes it to remain stationary relative to the generator's motion with relative displacement $z(t)$. While work is being done against the damping force in the mechanical damper, power that dissipates into the damper represents the generator output power. In particular, the VDRG is characterized by a damping force proportional to the relative velocity of the proof mass $d z(t) / d t$.

We must utilize several important parameters while carrying out simulation analysis with measured acceleration signals and the harvester model: the value of the proof mass $m$, spring constant $K$, damping coefficient $D$, and internal travel limit $Z_{\max }$. Of these variables, the proof mass $m$ and the internal travel limit $Z_{\max }$ are limited to an acceptable fraction of the mass and size of the object into which the generator is incorporated. In our later analysis we explain our choice of $m$ and $Z_{\max }$ based on the objects we wish to power.

Previous studies on inertial generators have shown that the maximum output power of a generator is proportional to the value of the proof mass independent of the shape of input acceleration waveform. The spring constant is chosen so that the generator resonates at some optimal frequency component $f$ such that a small bandwidth including $f$ maximizes the amount of energy recovered from the complex vibration spectrum. Accordingly, $K$ is calculated from the equation $2 \pi f=\sqrt{K / m}$ in terms of the selected proof mass $m$ and resonant frequency $f$. $Z_{\max }$ represents the maximum travel length of the proof mass inside the inertial generator. As previously mentioned, $Z_{\max }$ strongly depends on the size of the physical object into which the power harvester will be incorporated. A similar limitation is imposed on estimating the value of the proof mass.

The damping coefficient $D$ is related to the $Q$ factor, which refers to the ratio of the frequency at which the system oscillates to the rate at which the system dissi- 


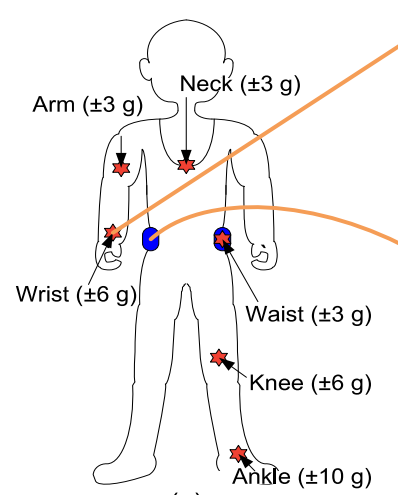

(a)

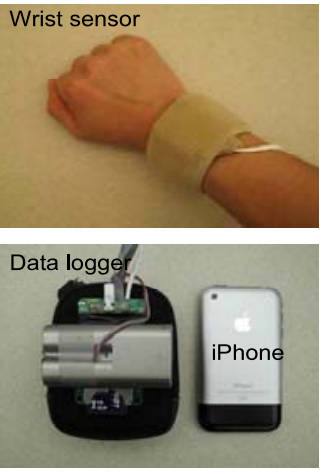

(b)
Figure 2. Figure (a) illustrates our experimental setup of six three-axis accelerometer modules and two data loggers mounted on body locations. Each accelerometer module was carefully chosen to match the probable range of acceleration in the corresponding body location. Figure (b) shows an accelerometer module mounted on the wrist and a data logger unit.

pates its energy. Generally, $D$ is chosen to be the lowest value that keeps the proof mass from reaching the internal travel limit $Z_{\max }$ of the generator. In our simulation, supposing $D$ to be fixed in practice by the generator mechanism, we searched for the optimal $D$ that resulted in the maximum generated power over the course of each day for each subject. Of course a more complex dynamically-damped generator could be imagined, and this generator's control system could be incorporated into the numerical model to more accurately estimate achievable power for any given generator.

\section{DATA COLLECTION AND ANALYSIS}

\section{Experimental Setup}

To acquire acceleration signals from six body locations of human subjects for an entire day, we have developed wearable data collection units comprising data loggers, accelerometer modules, and a lithium-ion camcorder battery $(0.1 \mathrm{Kg})$. Each data collection unit consists of two Logomatic Serial SD data loggers $(65 \mathrm{~mm} \times 110 \mathrm{~mm})$ from SparkFun Electronics, whose analog inputs are connected to six tri-axial accelerometer modules based on the ADXL330 $( \pm 3 g)$ and ADXL210 $( \pm 10 g)$ accelerometers from Analog Devices, and the MMA7260Q $( \pm 6 g)$ accelerometer from Freescale Semiconductor. Each accelerometer module is packaged in a small container custom-fabricated with a $3 \mathrm{D}$ printer and filled with hot glue to protect the sensor from dust or sweat. Thin, flexible wires interconnect each accelerometer module with the data logger. The data loggers are contained in a small waist-pack intended to hold a digital camera. The Logomatic SD data loggers are configured to record each analog input as a time series file on a $1 \mathrm{~GB}$ SD memory card, with a sampling rate of $80 \mathrm{~Hz}$. This configuration permits over 24 hours of continuous operation without the need to recharge the battery or exchange SD cards.

Our experiments consisted of capturing acceleration datasets from eight participants (four men and four women) wearing the data collection unit for three days (two weekdays and one weekend day). During this time, accel-

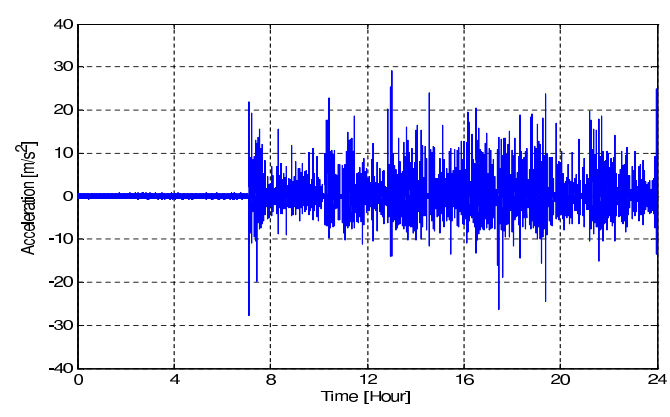

Figure 3. An example (the arm) of a filtered acceleration waveform over a 24 hours period.

eration was continuously recorded from six body locations where power harvesters could plausibly be mounted: around the neck, the arm, the wrist, the waist, the knee, and the ankle (see Figure 2-a). The neck accelerometer was worn around the neck on a lanyard, like a pendant, and the waist accelerometer was attached on the data logger to function like a cell phone holster on the waist belt. All the other accelerometers were fastened to the body using an elastic band with Velcro ends, as shown in Figure 2-b, except the knee sensor, which was fixed to the skin by a medical bandage because the elastic band on the knee often slid down while the participant was walking. The participants had the option to take off the data collection unit for certain parts of the day if they needed to (e.g., sleeping or taking a shower). For analysis on the variation of generated power during different activities, all the subjects were asked to record their activities and time on diary sheets.

\section{Processing of Acceleration Signals}

The acceleration time series we obtain from the bodymounted accelerometers corresponds to the acceleration experienced by the microscopic proof masses inside each accelerometer unit. Since we use 3-axis accelerometers, we record each orthogonal component of this acceleration separately, in $X, Y$, and $Z$. These axis labels are local to the accelerometer's reference frame because each accelerometer is attached to a different body part, and people move around constantly during the day. Fortunately, the reference frame of the accelerometer proof mass is the same as that of a proof mass inside a VDRG mounted at the same location. To obtain the displacements experienced by the accelerometers, for each axis a time series of differences in displacement of each accelerometer is first obtained by double-integrating the acceleration dataset. This displacement time series is then used to calculate the relative motion of the proof mass inside the VDRG by feeding the differences in displacement into the power harvester model.

It is important to note that we measured acceleration data with the assumption that we would harvest energy from daily human activities using free motion; that is, our VDRG does not experience forces beyond those due to its relative displacement and the inertia of its proof mass. For example, the accelerometers do not measure, and a VDRG would not harvest energy from, torque gen- 


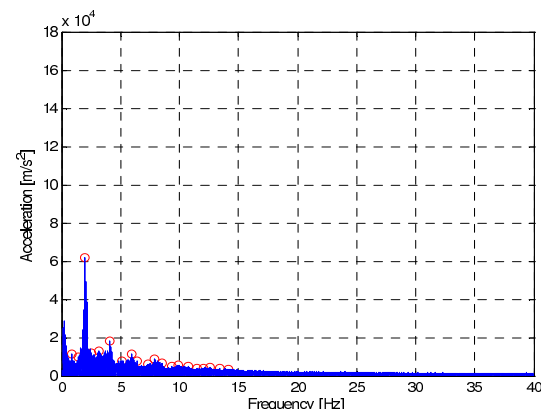

(a) neck

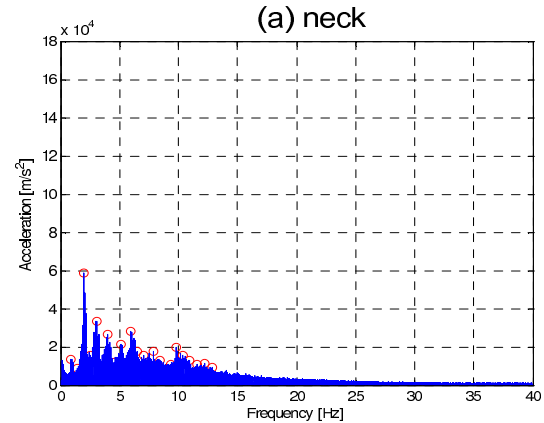

(d) waist

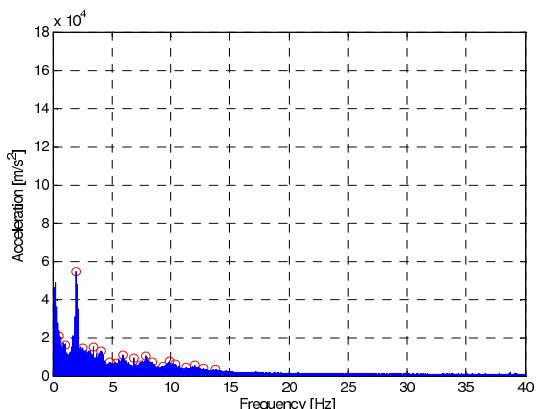

(b) arm

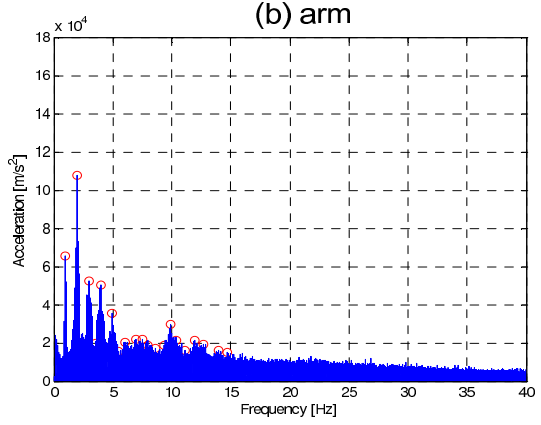

(e) knee

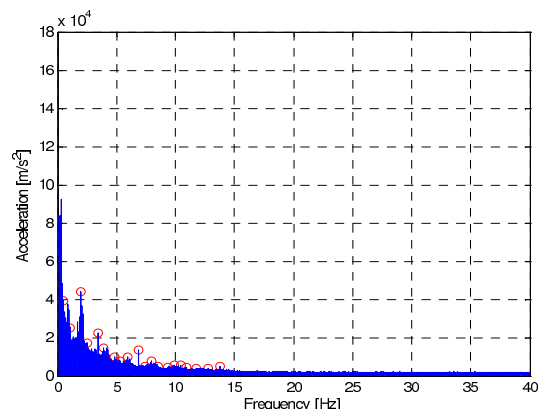

(c) wrist

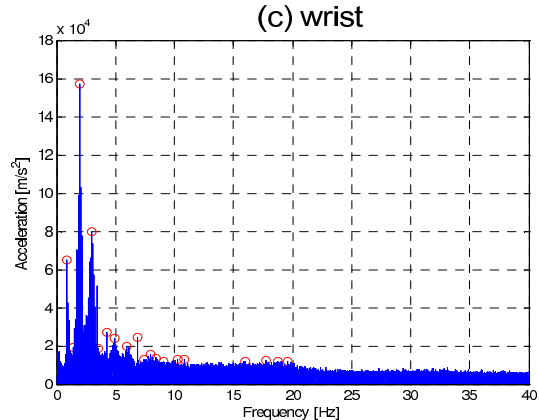

(f) ankle

Figure 4. The spectra of the acceleration signals from six locations.

erated while maneuvering a steering wheel to drive a car or pedaling a bicycle. Similarly, the ground reaction force on the feet while one is walking or running is not considered. This force-driven (or torque-driven) energy could be investigated with another type of sensor and power harvester [15, 27]. Because they add resistance to the body's motion beyond their own weight, these force- or torque-driven harvesters are not inertial in nature. In this work we consider only kinetic energy generated from human motion as an absolute magnitude of acceleration, assuming the mass of each part of the torso and the limb are held constant for each subject. In the case of the neck and waist accelerometers, although the movement of each sensor is not exactly the motion of the subjects, they do behave like real objects people wear (e.g., pendants or pouches).

Even though the data sheets for the accelerometers that we used provide typical zero-gravity output voltage and sensitivity (volt/gravity), manual calibrations were performed for all accelerometers to obtain more accurate translation from the voltage output of each accelerometer to its acceleration value. To accomplish this calibration, the $0 g$ voltage and sensitivity for every axis of each accelerometer were recorded by positioning each sensitive axis $(X, Y, Z)$ of the accelerometer at $+g$ and $-g$. Because accelerometers measure the static acceleration of gravity $(1 g)$ as well as the dynamic acceleration generated by pure human motion, gravity is always included in the measured acceleration data as a DC component. To eliminate the background signal (DC component) due to gravity, we high-pass filtered the measured acceleration signals with a $0.05 \mathrm{~Hz}$ cutoff frequency. Figure 3 shows an example of the filtered acceleration waveform.

\section{Data Analysis in Frequency Domain}

To gain more insight about the measured acceleration data, we generated frequency domain spectra by performing a FFT (Fast Fourier Transform) on the acceleration signal after removing the background signal due to gravity. Figure 4 displays the spectra of the acceleration signals from six locations on the body of one subject during one day. Since each plot is made with the same scale, we immediately observe that the lower body (waist, knee, and ankle) experiences much more pronounced acceleration than the upper body (neck, arm, and wrist). This suggests that the lower body is likely to yield more electrical energy converted from kinetic energy than the upper body. However, the upper body spectra (especially the wrist) contain energy at lower frequencies (below 0.5 $\mathrm{Hz}$ ) when compared to the lower body, probably because the upper body has a higher degree of freedom when moving than the lower body (e.g., talking while gesturing while sitting on a chair).

Although ordinary human motion is not a pure sinusoidal driving signal, i.e., $\cos (2 \pi f t)$, several large peaks appear in each spectrum. Accordingly, ordinary human motion can be approximated as a combination of several dominant frequency components. This observation leads us to the potential of designing an optimal inertial power harvester that can be accurately tuned to a selected dominant frequency range for a given set of ordinary human activities. We observe that the largest peak in each spectrum occurs at approximately 1 or $2 \mathrm{~Hz}$, which is as expected when we consider that the normal human walking pace is approximately two foot-falls per second.

To algorithmically determine the dominant frequency of each subject's activities throughout the day, we searched for the top 20 frequency components, ranked in order of observed energy from highest to lowest, from each 


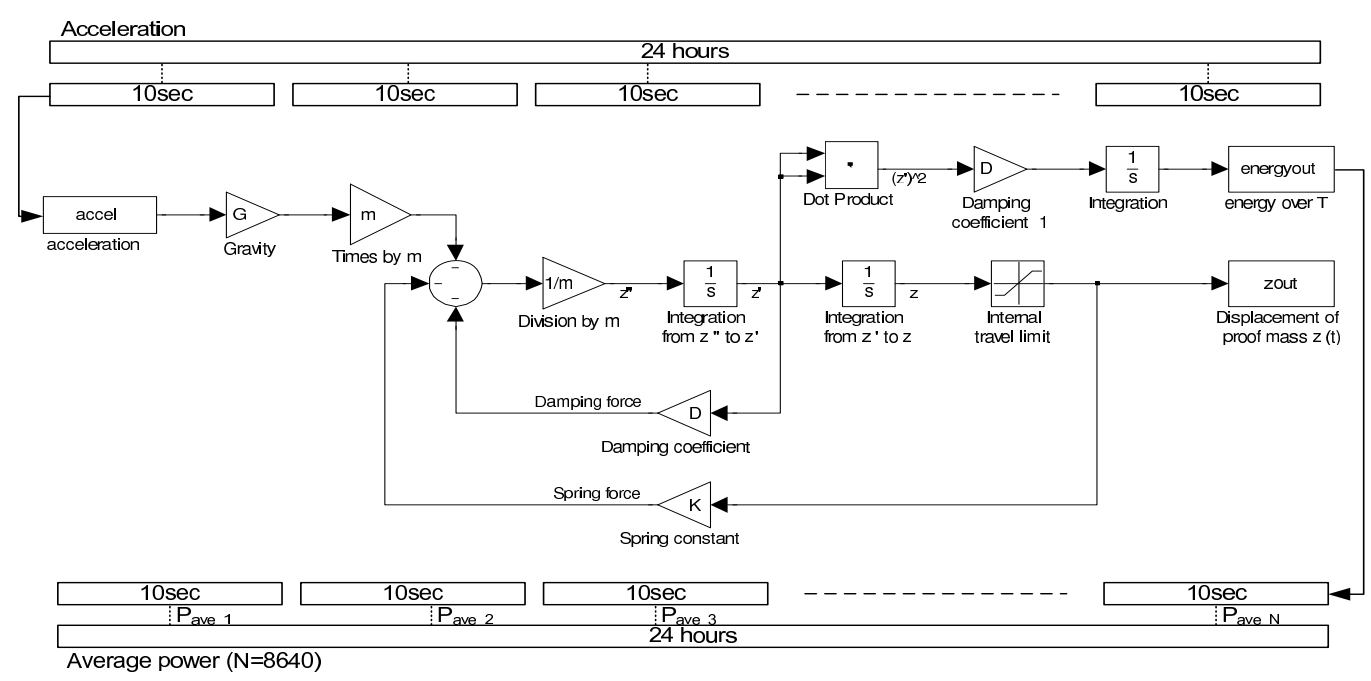

Figure 5. A flow chart of the signal processing pipeline for the power estimation procedure.

spectrum. In Figure 4, red circles depict the top 20 frequency components between $0.5 \mathrm{~Hz}$ and $40 \mathrm{~Hz}$. We neglect frequency components below $0.5 \mathrm{~Hz}$ due to the impracticality of manufacturing VDRGs with acceptable efficiency at such low frequencies. Although the top 20 frequency components may be considered dominant frequencies, perfectly sharp peaks are not observed because ordinary human motion is not sinusoidal. Accordingly, instead of treating the observed accelerations as pure tones, we need to investigate which frequency bins contain considerable kinetic energy to set the resonant frequency of the VDRG. Consequently, from the top 20 frequency components, we again sorted to obtain the 20 frequency bins by aggregating the adjacent frequency bins and ranking the sums. The top 20 frequency bin values are then used to tune the VDRG model to obtain an optimal inertial generator that yields the maximum output power with a measured acceleration signal. This optimization is performed by adjusting spring constant $K$ to satisfy the equation $2 \pi f=\sqrt{K / m}$, where $m$ is the value of the proof mass of the inertial generator and $f$ is the resonant frequency.

\section{SIMULATION AND RESULT}

\section{Simulation Setup}

To estimate the electrical power that could be generated by VDRGs incorporated into everyday electronic devices, we chose a wristwatch, a cell phone, and a shoe as representatives of typical mobile devices. A wristwatch is a good example of a device for which power harvesting technology has already been developed. Cell phones are common mobile devices we use every day, and their size and mass are similar to those of an MP3 player such as an iPod. Although a shoe is not per se a device that consumes electrical power, it does represent an object into which power harvesting technology can be embedded. A number of inventors have developed prototype shoe-mounted devices and described how they may be powered by energy harvesting, though most of those harvesters are of the non-inertial type that rely on inserting the harvester between the foot and the ground.
Based on an informal empirical survey of digital devices, and some general assumptions, we have developed what we believe are reasonable values of the proof mass $m$ and internal travel length $Z_{\max }$ for a harvester incorporated into each object: $2 \mathrm{~g} / 4.2 \mathrm{~cm}, 36 \mathrm{~g} / 10 \mathrm{~cm}$, and 100 $\mathrm{g} / 20 \mathrm{~cm}$ for a wristwatch, a cell phone, and a shoe, respectively. The proof mass of $2 \mathrm{~g}$ for the wristwatch was derived from the mass of a commercialized product [22] and the interval travel length for a wristwatch was obtained from the average value of the diameters of 41 SWATCH wristwatches; the proof mass of the cell phone was derived from one-third of the average mass of 40 NOKIA cell phones; and the proof mass of the shoe was obtained from an assumption that shoe length and mass for the normal adult would be greater than $20 \mathrm{~cm}$ and $300 \mathrm{~g}$, respectively.

Based on the chosen objects and the body locations, we measured accelerations and selected the power harvester configuration for simulation, displayed in Table 2. We could not imagine any useful digital object mountable on the knee, so we excluded this case from the simulation. We considered all other configurations as plausible conditions in daily life. The wristwatch cases represented either a pendant worn around the neck or a watch worn on a wrist; the cell phone cases represented a phone suspended on a necklace around the neck, inside an arm band, or in a waist holster; and the shoe-size object represented a device that could be worn on the ankle since such devices would not typically be placed on other parts of the body.

\section{Power Estimation Procedure}

We have developed the following procedure for using the measured acceleration dataset, in combination with the VDRG model, to estimate available power as illustrated in Figure 5. First, an acceleration data set for an entire day is divided into $8640(=24 \mathrm{hr} * 60 \mathrm{~min} / \mathrm{hr} * 60 \mathrm{sec} / \mathrm{min} *$ $1 / 10 \mathrm{sec}$ ) ten-second interval fragments. Each fragment is fed into the generator model so that the displacement of the generator frame (i.e., the accelerometer container) $y(t)$ can be obtained by double integrating the accelera- 
Table 2. Power harvester configuration used for simulation.

\begin{tabular}{|c|c|c|c|}
\hline & Wristwatch & Cell Phone & Shoe \\
\hline Neck & $\circ$ & $\circ$ & \\
\hline Arm & & $\circ$ & \\
\hline Wrist & $\circ$ & & \\
\hline Waist & & $\circ$ & \\
\hline Knee & & & \\
\hline Ankle & & & $\circ$ \\
\hline
\end{tabular}

tion signal. Subsequently, the relative displacement $z(t)$ and derivative $d z(t) / d t$ of the proof mass can be calculated from $y(t)$. As mentioned in the previous section, the electrical power generated in the inertial generators can be represented by the power dissipated in the mechanical damper shown in Figure 1. Accordingly, the energy dissipated in the damper, $E_{d}$ is:

$$
E_{d}=\int_{Z_{1}}^{Z_{2}} F \cdot d z
$$

where $F=D \dot{z}$ is the damping force, and $Z_{1}$ and $Z_{2}$ are the start and end positions, respectively, for the line integral. Equation 2 can be expanded as follows:

$$
E_{d}=\int_{Z_{1}}^{Z_{2}} D \dot{z} \cdot d z=\int_{t=0}^{T} D \frac{d z}{d t} \cdot \frac{d z}{d t} d t=D \int_{t=0}^{T}(\dot{z})^{2} d t
$$

The average power during time interval $T=10 \mathrm{sec}$ is

$$
P_{\text {average }}=\frac{1}{T} E_{d}=\frac{D}{T} \int_{t=0}^{T}(\dot{z})^{2} d t
$$

By applying this procedure to all fragments successively, the average power value for every ten-second fragment is obtained. The power calculation procedure has been implemented together with the VDRG model in MATLAB Simulink.

Because the accelerometer modules we deployed in our experiments recorded the accelerations along $X, Y$, and $Z$ axes, we assumed that a practical VDRG unit would be built with a single axis in alignment with one of the axes of the accelerometers. The preferred orientation of the VDRG is then chosen based on which one of the three accelerometer axes yields the maximum output power.

\section{Optimization and Result}

Given $m$ and $Z_{\max }$ as derived for each of the selected objects, and $K$ chosen based on the FFT analysis for each configuration in Table 2, we optimized the power harvesters by searching for the optimal damping coefficient $D$, which maximizes average output power $P$. $P$ then represents the energy generated while the subjects wore the data collection unit over the entire measurement time. First, we searched for three different $D$ values in terms of three $K$ calculated from FFT analysis for three days for each of the eight subjects. Subsequently, we found one optimal $D_{o}$ for three days for each subject using one dominant frequency $f_{o}$ or spring constant $K_{o}$, which can be obtained by concatenating all acceleration data for three days and performing the same method described in the FFT analysis section.
Table 3. Generated output power: C1-a watch hanging on the neck, C2-a watch on the wrist, C3-a phone hanging on the neck, C4-a phone on the arm, C5-a phone on the waist, and C6-a shoe on ankle

\begin{tabular}{|c|r|r|r|r|r|r|}
\hline Sub & $\begin{array}{r}\text { C1 } \\
(\mu \mathrm{W})\end{array}$ & $\begin{array}{r}\mathrm{C} 2 \\
(\mu \mathrm{W})\end{array}$ & $\begin{array}{r}\mathrm{C} 3 \\
(\mathrm{~mW})\end{array}$ & $\begin{array}{r}\mathrm{C} 4 \\
(\mathrm{~mW})\end{array}$ & $\begin{array}{r}\mathrm{C} 5 \\
(\mathrm{~mW})\end{array}$ & $\begin{array}{r}\mathrm{C} 6 \\
(\mathrm{~mW})\end{array}$ \\
\hline \multirow{3}{*}{1} & 14 & 164 & 0.2 & 1.1 & 0.1 & 2.8 \\
& 31 & 265 & 0.6 & 1.8 & 0.1 & 4.2 \\
& 25 & 195 & 0.7 & 1.7 & 1.4 & 12.6 \\
\hline \multirow{3}{*}{2} & 48 & 167 & 0.3 & 1.4 & 0.1 & 2.8 \\
& 35 & 162 & 0.4 & 1.4 & 0.2 & 2.9 \\
& 10 & 67 & 0.1 & 0.7 & 0.2 & 2.6 \\
\hline \multirow{3}{*}{3} & 16 & 127 & 0.4 & 1.2 & 0.5 & 6.0 \\
& 26 & 137 & 0.2 & 1.3 & 0.2 & 2.3 \\
& 16 & 153 & 0.2 & 1.3 & 0.4 & 3.4 \\
\hline \multirow{3}{*}{4} & 8 & 57 & 0.3 & 0.5 & 0.3 & 2.6 \\
& 13 & 66 & 0.4 & 0.7 & 0.3 & 3.1 \\
& 12 & 84 & 0.5 & 0.7 & 0.4 & 4.0 \\
\hline \multirow{3}{*}{5} & 20 & 141 & 0.7 & 0.6 & 0.6 & 10.5 \\
& 19 & 143 & 0.7 & 0.6 & 0.6 & 11.5 \\
& 36 & 170 & 1.2 & 1.1 & 1.4 & 12.0 \\
\hline \multirow{3}{*}{6} & 37 & 130 & 0.7 & 0.6 & 0.1 & 1.0 \\
& 37 & 143 & 0.7 & 0.7 & 0.2 & 10.4 \\
& 73 & 225 & 1.4 & 1.3 & 0.6 & 3.1 \\
\hline \multirow{3}{*}{7} & 10 & 54 & 0.1 & 0.3 & 0.1 & 3.1 \\
& 18 & 96 & 0.7 & 0.5 & 0.7 & 4.1 \\
& 30 & 322 & 0.3 & 0.9 & 0.3 & 0.7 \\
\hline \multirow{3}{*}{8} & 32 & 144 & 0.2 & 0.7 & 0.5 & 3.7 \\
& 29 & 176 & 0.2 & 1.2 & 0.3 & 1.8 \\
& 99 & 346 & 1.7 & 2.1 & 0.8 & 6.5 \\
\hline
\end{tabular}

With the harvesters optimized for the given configuration for each of our subjects, we are able to estimate the electrical power for a given form factor. However, even though the harvesters have been theoretically optimized, other practical questions may need to be addressed. First, we must estimate the expected mechanicalto-electrical conversion efficiency of the harvester, in terms of useful electrical power obtained as a fraction of the amount of power dissipated in the damper. Although the dissipated power represents the generated power of the harvester in Figure 1, the two values might differ due to the mechanical aspects of implementation and electrical circuitry efficiency. Therefore, by applying the characteristics of the PMG 27 [5] harvester to our simulation method and then comparing the result to the data sheet, we estimated that typical mechanical to electrical conversion efficiency would be around $20 \%$. Consequently, we assumed that only $20 \%$ of the energy dissipated in the harvester's damper would be provided to the electronics it was powering. Second, we assumed that all energy generated over the day could be stored in some form of storage and would be used to power wearable electronics. Our last assumption is that the direction of the power harvester in real objects could be continuously aligned with the axis that generates the maximum output for a whole day. Based on these assumptions and the simulation results, the average electrical power expected from a VDRG embedded in each object was 155 $\pm 106 \mu \mathrm{W}, 1.01 \pm 0.46 \mathrm{~mW}$, and $4.9 \pm 3.63 \mathrm{~mW}$ for the wristwatch, the cell phone, and the shoe, respectively, 


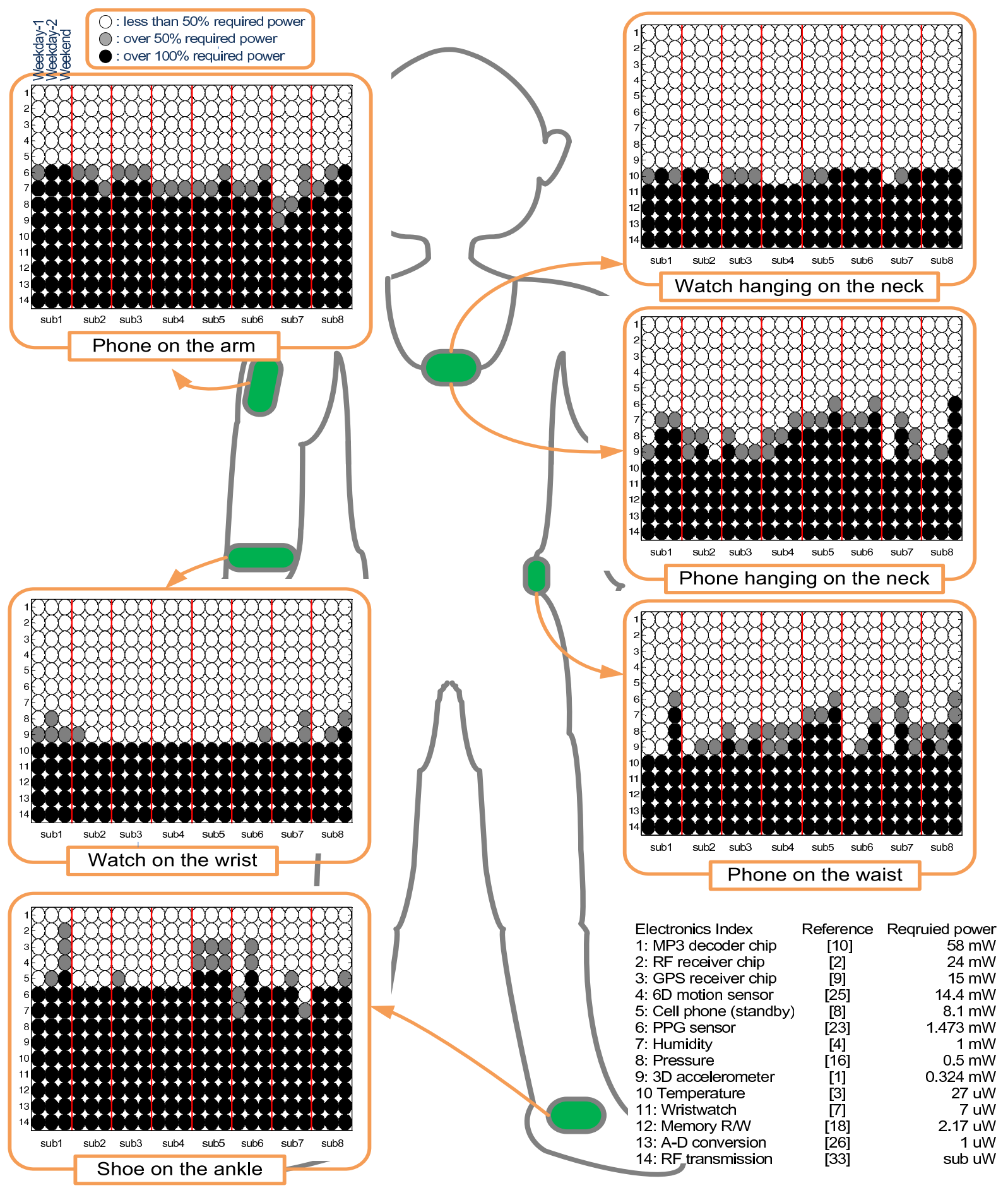

Figure 6. The ratio of the electrical output power of the harvester to the required power for wearable electronics: a watch hanging on the neck, a watch on the wrist, a phone hanging on the neck, a phone on the waist, a phone on the arm, and a shoe on the ankle. The $\mathrm{X}$-index represents the subjects ( 8 subjects $* 3$ days $=24$ days), and the $\mathrm{Y}$-index represents the wearable electronics considered in Table 1. 
depending upon the body location on which the power harvester was mounted. Table 3 illustrates the generated power for our eight subjects.

\section{DISCUSSION}

From the results just presented, we can determine whether the wearable electronics in Table 1 can be powered for a given subject on a given day, depending upon the simulation conditions shown in Table 2. Figure 6 shows the result of using the garnered output power to power different wearable electronics at various parts on the body. In a number of cases, the output power is insufficient to continuously power the highest demanding electronics such as the MP3 decoder chip, the RF receiver chip, and the GPS receiver chip. However, the VDRG can be used to charge a battery for intermittent operation of those devices. One can imagine using the garnered power to charge a back-up battery when a standard recharging power source is not available. Low-power electronics such as the medical telemetry devices and wristwatches can be powered continuously. If we consider a simplified model of a signal processing pipeline for a sensor-enabled system consisting of a collection of sensors, an analog to digital converter, micro-controller, and short-range wireless transmission, our results indicate that we are able to continuously power these types of integrated systems. As new low-power technologies emerge, we can imagine many more senors being able to be supported by this harvesting approach. Thus, we begin to see the feasibility of developing wearable electronics powered from normal everyday activities.

Although the diary sheets that the participants recorded while collecting data do not perfectly express all their activities, it is still valuable to look into the generated power in terms of some of the activities. Figure 7 shows the power generated over 24 hours for subject 1 with the activities annotated. In this figure, the available power at the upper arm shows that a phone-scale harvester on the arm will generate about $15 \mathrm{~mW}$ while running. Thus, our specific ultra-low power GPS chip can be powered, even though much more power might be necessary to operate extra components such as an LCD display. Likewise, a shoe harvester located at the ankle will produce $20 \mathrm{~mW}$ enough to power the selected GPS chip, but still insufficient for an entire GPS unit. The figure for power on the wrist shows the continuous availability of more than $60 \mu \mathrm{W}$ power over nearly all measurements, because of the large number of ambulatory activities taking place over the course of the day. Similarly, arm power shows very high power generation during activities in which the arms are moved vigorously.

\section{LIMITATIONS AND FUTURE WORK}

Although our results show that some electronics cannot be continuously powered by the type of unobtrusive VDRG-based harvester that we have studied, the apparent power shortage could be mitigated in several ways. First, because the output power of the harvester is proportional to the value of the proof mass regardless of the acceleration waveform and the architecture of the harvester, we can increase the output power with a heavier proof mass in the harvester. But overwork and fatigue,

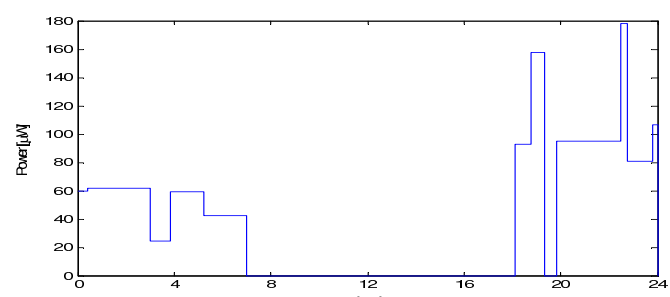

(a)

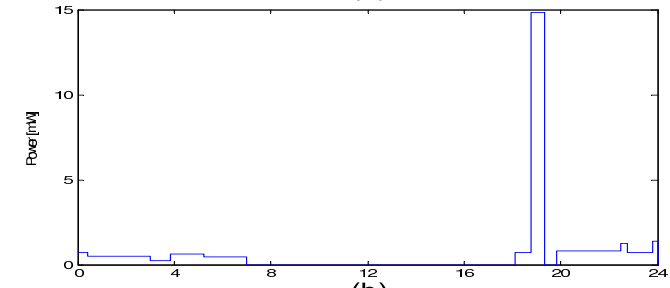

(b)

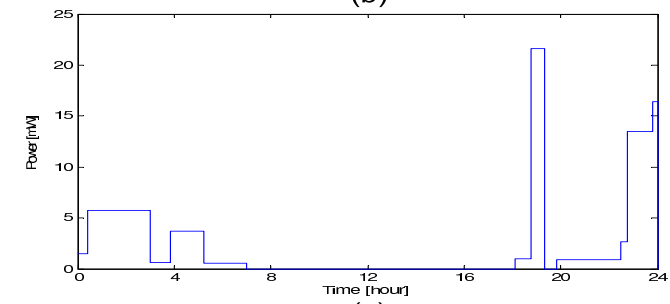

(c)

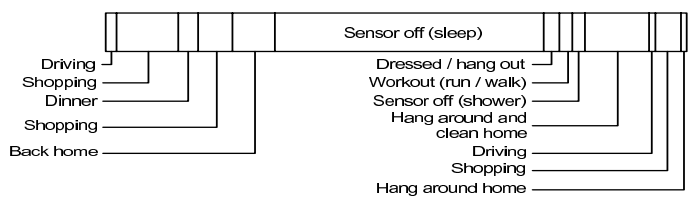

(d)

Figure 7. Power generated with a watch on the wrist, a phone on the arm, and a shoe on the ankle Friday through Saturday afternoon for the subject 1. (a) watch on wrist, (b) phone on arm, (c) shoe on ankle, (d) activity annotation.

resulting from too much weight, would have to be carefully considered while increasing the proof mass. Second, we may use two other axes as well as the axis that generates the maximum energy in designing the power harvester, i.e., a harvester with three mass-spring systems aligned with the $X, Y$, and $Z$ axes. Thus, powering a GPS receiver is probably realizable, assuming that an individual is hiking on a mountain wearing mountainclimbing boots into which a heavier proof mass or three mass-spring systems are embedded.

Although we have optimized the harvester for a given subject and location, a harvester with a single $K / D$ combination could be designed for a given location that would work well enough for all of our subjects. The method would be analogous to that used in optimizing the harvester for a given individual. We can first concatenate the data of all the days into a single time series, which may form a huge data set; then we can perform FFT analysis; and finally we might be able to obtain a single dominant frequency for a given location. After optimizing $D$ with the single $K$ value, a harvester for all subjects can be designed for given locations although some variation would obviously appear in the performance of the harvester across subjects. 
We expect that a typical VDRG harvester will be implemented with a permanent magnet (serving as the proof mass) moving through a stationary coil. Due to Lenz's law, the damping force of the harvester can be adjusted by changing the effective load resistance presented to the generator coil. This variable-load-impedance method permits dynamic control of the damping constant $D$. It could be implemented with either an analog or a digital control system, though probably a digital control system using a pulse width modulation technique to dump energy into a storage capacitor or battery for a certain portion of the proof mass travel would be the most straightforward implementation. Similarly, if spring constant $K$ of the spring suspending the proof mass in a harvester is adjustable mechanically or electrically, a dynamically tunable harvester that could adapt $K$ and $D$ can be realized to maximize the output power in terms of the individual, the activity, and the form factor. We expect this avenue of adaptive tuning of the VDRG to represent a significant and intriguing area for future work.

\section{CONCLUSIONS}

We have presented the first 24-hour in situ, continuous study of inertial power harvester performance from everyday activities. We discussed the design of a specific type of inertial harvester, know as a velocity damped resonant generator (VDRG). Based on acceleration data collected from six body locations from each of our eight different participants, we analyzed the amount of energy that can be garnered at those locations. Our findings show that is it feasible to continuously operate motionpowered wireless health sensors and that activity-specific inertial power harvesting will provide continuous operating power for some commonly-used consumer electronics devices, such as a GPS receiver. We also found that motion-generated power can also provide an intermittent power source for more power intensive devices like a MP3 player or cell phone. With the advent of new low-power integrated circuits and sensors, or the development of adaptively tuned VDRG, we can imagine supporting a larger number of devices in the near future.

\section{REFERENCES}

1. ADXL330 3-axis accelerometer, http://www.analog.com/ en/prod/0,2877,ADXL330,00.html.

2. ASK single conversion receiver TDA 5200, http://www. infineon.com/dgdl/TDA5200_V2+9.pdf?folderId= db3a304412b407950112b408e8c90004\&fileId= db3a304412b407950112b411c7db176e.

3. FM20 low-voltage analog temp sensor, http://www. fairchildsemi.com/pf/FM/FM20.html.

4. HIH-3610 series, http://content.honeywell.com/sensing/ prodinfo/humiditymoisture/009012_2.pdf

5. PMG $\mu$-generator, http://www.perpetuum.co.uk/.

6. VEH-360 electromechanical generator, http://www.ferrosi. com/energy-harvesters.html.

7. Seiko AGS quartz watch, http://www.epson.co.jp/e/ company/milestones/19_ags.pdf, 1998.

8. Cell phone power management requires small regulators with fast response, http://www.commsdesign.com/printableArticle/ ?articleID=12801994, 2002.

9. GNR1040 GPS receiver, http://www.glonavgps.com/images/ glonav_gnr1040_pb.pdf, 2004.
10. Samsung Helix, Nexus and Pioneer Inno power consumption, http://www.orbitcast.com/archives/samsung-helix-nexus-andpioneer-inno-po wer-consumption.html, 2006.

11. R. Amirtharajah and A. Chandrakasan. Self-powered signal processing using vibration-based power generation. IEEE J. Solid-State Circuits, 33:687-695, 1998.

12. N. Bharatula, S. Ossevoort, M. Stäger, and G. Tröster. Towards wearable autonomous microsystems. In Pervasive04, pages 225-237, 2004.

13. T. Büren, P. Mitcheson, T. Green, E. Yeatman, A. Homes, and G. Tröster. Optimization of inertial micropower generators for human walking motion. IEEE J. Sensors, 6(1):28-38, 2006.

14. A. Chapuis and E. Jaquet. The History of the Self-Winding Watch. Roto-Sadag, 1956.

15. J. Donelan, Q. Li, V. Naing, J. Hoffer, D. Weber, and A. Kuo. Biomechanical energy harvesting: Generating electricity during walking with minimal user effort. Science, 319(5864):807 - 810, 2008.

16. C. Hierolda. Low power integrated pressure sensor system for medical applications. Sensors and Actuators A: Physical, 73(1-2):1261-1265, 1999

17. W. Huynh, J. Dittmer, and A. Alivisatos. Hybrid NanorodPolymer solar cells. Science, 295(5564):2425-2427, 2002.

18. G. Mathur, P. Desnoyers, D. Ganesan, and P. Shenoy. Ultra-low power data storage for sensor networks. In IPSN06, pages 374-381, 2006.

19. P. Mitcheson, T. Green, E. Yeatman, and A. Holmes. Architectures for vibration-driven micropower generators. IEEE J. MEMS, 13(3):429-440, 2004.

20. J. Paradiso. Systems for human-powered mobile computing. In DAC06, pages 645-650, 2006.

21. J. Paradiso and M. Feldmeier. A compact, wireless, self-powered pushbutton controller. In Ubicomp01, pages 299-304, 2001.

22. J. Paradiso and T. Starner. Energy scavenging for mobile and wireless electronics. IEEE Pervasive Comp., 4(1):18-27, 2005.

23. S. Rhee, B.-H. Yang, and H. Asada. Artifact-resistant, power-efficient design of finger-ring plethysmographic sensors part i:Design and analysis. In EMBS00, pages 2792-2795, 2000.

24. L. Rome, L. Flynn, E. Goldman, and T. Yoo. Generating electricity while walking with loads. Science, 309(5741):1725-1728, 2005.

25. A. Sadat, H. Qu, C. Yu, J. S. Yuan, and H. Xie. Low-power CMOS wireless MEMS motion sensor for physiological activity monitoring. IEEE Trans. Circuits Syst, 52(12):2539-2551, 2005.

26. J. Sauerbrey, S. Landsiedel, and R. Thewes. A $0.5-\mathrm{v} 1-\mu \mathrm{w}$ successive approximation ADC. IEEE J. Solid-State Circuits, 38(7):1261-1265, 2003

27. N. Shenck and J. Paradiso. Energy scavenging with shoe-mounted piezoelectrics. IEEE Micro, 21(3):30-42, 2001.

28. T. Starner. Human-powered wearable computing. IBM Systems Journal, 35(3\&4):618-629, 1996.

29. T. Starner and J. Paradiso. Human-generated power for mobile electronics. Low-Power Electronics Design, CRC Press, chapter 45, pages 1-35, 2004.

30. M. Strasser. Miniaturized thermoelectric generators based on poly-Si and poly-SiGe surface micromachining. Sensors and Actuators A: Physical, 97-98:535-542, 2002.

31. C. Williams and R. Yates. Analysis of a micro-electric generator for microsystems. Sensors and Actuators A: Physical, 52:8-11, 1996.

32. D. Yates and A. Holmes. Micro power radio module. ORESTEIA IST project, 2003.

33. E. Yeatman. Advances in power sources for wireless sensor nodes. In BSN06, pages 20-21, 2004. 